\title{
Selected Experiences with Optimization Tests of the Kaplan-Type Hydraulic Turbines
}

\author{
Adam Adamkowski ${ }^{1}$, Mariusz Lewandowski ${ }^{1}$ and Stanisław Lewandowski ${ }^{2}$ \\ 1. The Szewalski Institute of Fluid-Flow Machinery, Polish Academy of Sciences, Gdańsk 80-952, Poland \\ 2. Lewandowski Stanistaw, HydroConsult, ul. Glińskiego 13, Bolszewo 84-239, Poland
}

Received: January 09, 2014 / Accepted: February 13, 2014 / Published: June 30, 2014.

\begin{abstract}
The paper presents selected experience of the authors resulting from the optimization tests of double-regulated water turbines. Among the methods for measuring the discharge through the turbine used in such tests, particular attention was paid to index methods allowing to measure the relative discharge through the turbine-the index current meter method and the methods based on measuring the differential pressure between two points properly located at the turbine flow system (i.e., Winter-Kennedy method). These methods contribute to effective reduction of the cost of optimizing the turbine that is extremely important for small hydropower plants regarding installed capacity. The paper presents selected examples of the optimization tests and experiences that arise from these tests.
\end{abstract}

Key words: Double-regulated water turbines, cam dependence, flow measurement index methods, Winter-Kennedy index method, current meter index method.

\section{Introduction}

In recent years, issues connected with the optimization of hydropower plant operation have been pushed into the background of owners and users of hydropower plants interests. In current formal and legal conditions most hydro power plants, even those having possibility to accumulate water in the upper reservoir, are forced to work in a run-of-river system of operation. Environmental aspects and ambiguous provisions of the Water Framework Directive is a pretext to impose on hydropower plant users to maintain stability of the water level in the upper reservoir allowing only a small change of this level. In this situation, for hydropower plant users the only crucial operating parameter is the amount of the generated active power, regardless of the cost of its production resulting from the energy conversion efficiency of hydraulic energy into the

Corresponding author: Mariusz Lewandowski, Ph.D., research fields: hydraulic transients, vibration and efficiency tests of hydraulic turbines. E-mail: mlew@imp.gda.pl. electric energy. Energetic efficiency coefficient is becoming less important, because it does not translate easily to the amount of revenue from electricity production. This is the basic reason why the operation of hydro units with maximum possible efficiency is not a primary objective for hydropower plant users. As a consequence, the lack of attention to the proper operation of power control systems, especially in double regulated turbines like Kaplan turbines, is observed. Double regulation system in turbines used for an independent adjustment of the runner blades and wicket gate openings, allows the turbine to work with high efficiency in a wide range of load changes. The basic requirement for turbine operation at maximum efficiency under certain hydrological conditions (the disposable head of the plant and the required discharge through the turbine) is to adjust opening angles of the wicket gates and the runner blades according to so-called optimal cam dependence.

Operating of hydropower plants with the maximum efficiency enables the optimum utilization of disposable 
hydraulic potential that translates directly to maximum electricity production, as well as a significant reduction of the dynamic loads of constructional components of the hydrdrounit. This is due to:

- reduction of pressure pulsations in turbine flow system and dynamic loads of the components of this system;

- reduction of dynamic loads in the rotating system, in particular in bearing nodes of the machine (shaft guide bearings, pin bearings of the wicket gate, pin bearings of the runner blades, and bearing in kinematic regulation system);

- reduction of dynamic loads on structural elements of the hydraulic constructions;

- reduction of operation zones with excessive cavitation.

Unfortunately for users and operators of the hydropower plants, these issues are becoming important mainly in emergency situations or when the damage parts of hydro unit start to determine the amount of the power generation and the efficiency of the hydro unit production. But often, even in such situations only expeditious remedies involving the repair of damages, reconstruction or replacement of these parts are applied. Costly construction changes or deeper modernizations are very rare as they require much larger financial commitment. Even more rarely, optimization tests are carried out after such deep modernizations, which in turn should lead to the determination of new, optimal cam dependences. Power plant owners are not willing to invest in such tests, whose purpose is to determine and improve the indicators that do not directly translate into revenue derived from electricity production. In most cases, the costs of tests carried out to determine the absolute efficiency of the turbine using the methods for measurement of the absolute discharge through the machine cause such situation. Among hydropower plant users, the belief that measurements of absolute discharge are necessary to determine the optimal cam dependence is very common.
The authors of this paper show that for the optimization tests it is not necessary to measure the absolute discharge through the turbine and the relative value of the discharge is needed. Measurement methods that allow to determine the relative value of the discharge, are characterized by a significantly lower costs and time-consumption from methods for determining the absolute discharge. Choosing the appropriate method from among several available methods approved by relevant standards in this field, however, requires careful study of conditions and the proper preparation of the measuring systems [1-3].

Even the results from turbine model tests or CFD (computational fluid dynamic) simulations do not guarantee that the cam dependences determined basing on these results will be optimal in real conditions [4]. Some changes that can significantly affect the performance of machines are taking place during the operation of the power plant [5]. Modernization changes carried out during overhauls often affecting the operating parameters of the hydro units. Hence, there is the need to verify the cam dependence by performing specialized measurements in such situations. Such tests should be performed by the hydropower plant user every few years and, what is mandatory, after each overhaul and modernization of the turbine governor and every component associated with the regulation system. Ignoring these rules by owners and operators of hydropower plants leads to operating the hydro units far from their maximum efficiency. This is the reason for the lower electricity production than the production that can be achieved using disposable hydraulic potential. In addition, many important structural elements of hydropower plant operated in sub-optimal operating point are usually exposed to increased dynamic loads, resulting in accelerated and excessive wear. This situation leads to significant financial losses.

The experience of the authors of this paper shows that the efficiency of electricity generation in hydropower plants could increase by up to several 
percent only through optimization of operating hydro units consisting in setting the optimal cam dependences in the turbines governors. Lack of knowledge about modern methods of optimization tests causes that the power plant users consciously abandon these tests in order to achieve enigmatic savings. Therefore, the cam dependences in Kaplan-type turbines are very often significantly different from the optimal ones. This particularly concerns the hydro units, which were operated without verification of the cam dependence for a very long period of time. There are plenty of hydro units that from the beginning are operated without verification of cam dependence set in the governor by the producer of the machine. For the owners of hydropower plants, the main test giving the proof of the proper construction of the hydro unit is the level of maximum power achievable at a given head (often referred to the upper water level). However, it is more and more often that the effects of setting the properly verified cam dependences contribute to turning the attention of the hydropower plant users to issues connected with controlling the correctness of the turbine regulation system settings.

\section{Determining an Optimal Cam Dependence}

The primary objective of optimization tests is to determine the cam dependence, at which the tested machine reaches the maximum possible efficiency under given hydraulic conditions (head $H$ ). During these tests, several so-called propeller characteristics are determined at the subsequent runner blade openings covering the full range of their possible changes $[4,6$, 7]. Propeller characteristics are usually calculated as a relationship between the hydro unit efficiency $\eta$ and the discharge $Q$. Additionally, the relationship between the discharge and the wicket gate opening $Y_{w g}$. These parameters are measured for each propeller characteristic examined with constant opening angle of the runner blades $y_{r b}$ and several opening angles of the wicket gate opening. Besides discharge measurements during the tests, the head and the active power $P$ are also measured. Basing on these parameters, the hydrounit efficiency is determined. The obtained propeller curves $\left(\eta=\mathrm{f}(Q)\right.$ at $y_{r b}=$ const.) are used for determination of the envelope efficiency curve, i.e., a curve tangent to each of the propeller curves obtained. This envelope curve represents the optimal points of operation at a given head (at which the test was carried out) and for different loads (power $P$, discharge $Q$ ). On the basis of this envelope curve, the points with coordinates $\left(Y_{w g} ; y_{r b}\right)$ are obtained and they define the optimal cam dependence $y_{r b}=\mathrm{f}\left(Y_{w g}\right)$ for a given head. If the tested machine is normally operated in a wide range of head changes, it is often necessary to carry out optimization tests for several head values and then recalculate properly the optimal cam dependences for the whole range of head changes.

Optimization of hydro unit operation realized basing on the criterion of maximal efficiency ensures that cam dependence determined on the basis of these tests enable maximum utilization of disposable hydraulic potential. Setting turbine governor according to optimal cam dependences ensure operation of the turbine with maximal achievable efficiency at a given hydrological conditions and at a chosen power load.

The biggest problems that are encountered during the optimization tests are those associated with the measurement of discharge through the turbine. For discharge measurement, the primary methods are usually used. However, for the purposes of optimization tests the absolute value of discharge through the turbine is not necessary. Much easier to carry out and less expensive are measurements of the relative value of the discharge. The results of these measurements are relative efficiency values (efficiency index), but this has no significant effect on the quality of cam dependence determined basing on the optimization tests.

\section{Basic Techniques of Flow Measurements for Optimization Tests on Kaplan-Type Turbines}

As it was mentioned earlier, the optimization tests of the double-regulated turbines may be accomplished 
using the index efficiency values. The index efficiencies of the machine are calculated basing on the measured values of the specific hydraulic energy (head), along with the active power and flow rate values measured using uncalibrated methods. The relative efficiency is expressed by the ratio of any index efficiency to the reference index efficiency, for instance the maximal measured index efficiency.

Relative discharge (flow rate) values in such kind of tests can be determined using following methods:

The method of pressure difference between two points properly situated in the turbine spiral case-the Winter-Kennedy method;

The method of pressure difference is choosing from two properly selected points in bulb or tubular turbines.

Other methods of relative flow rate measurement include:

(1) The current meter method with a single (or several) propeller current meter located in the flow channel-called as the simplified index current meter method;

(2) The method of pressure difference between two properly situated sections in convergent segment of the turbine penstock;

(3) The centrifugal-head method-measuring pressure difference between the inner and outer part of the conduit elbow;

(4) The acoustic method, mainly using the single-path ultrasound flow meter.

The practical use of the above mentioned methods is less complicated and much cheaper than the principal method used for absolute discharge measurements.

Further in the paper principles and examples of application of the Winter-Kennedy method, the differential pressure and current meter index methods are presented.

\subsection{Methods Based on Measurement of Differential} Pressure

Winter-Kennedy method in classic Kaplan turbines and the differential pressure method in bulb turbines have similar theoretical foundations. In both methods, the flow is dependent on the measured pressure difference between two corresponding points located in flow system according to the formula:

$$
Q=K \cdot \Delta p^{n}
$$

where, $K$ is the constant coefficient, experimentally determined from calibration, while $n$ is power exponent, theoretically equal to 0.5 .

The Winter-Kennedy method utilizes the static pressure difference between the outer and inner side of the turbine spiral case due to the centrifugal force acting on the curved streams of liquid in the spiral case $\left(\Delta p=p_{1}-p_{2}\right)$. The possible location of the pressure taps is presented on Fig. 1a according to ISO 60041 Standard for a concrete half-spiral chamber of the classic Kaplan turbine.

A sample distribution of pressure taps of the differential pressure method in the case of flow system of the hydro-unit with a bulb turbine (without a spiral) is shown in Fig. $1 b$.

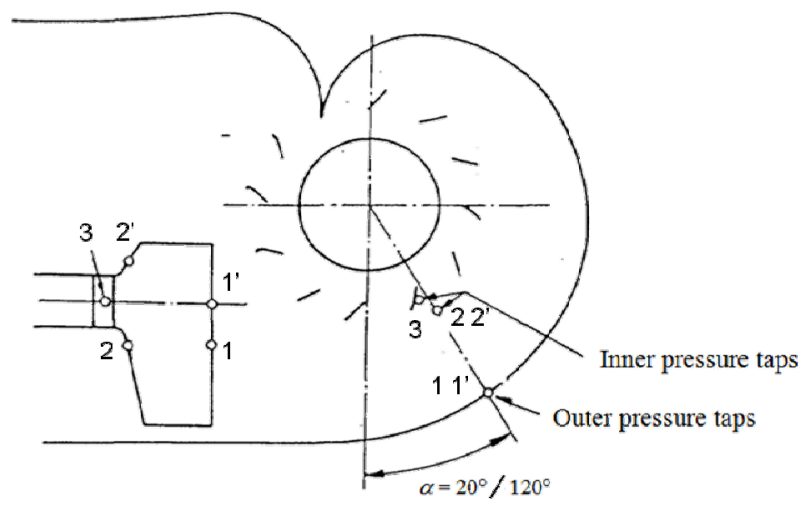

(a)

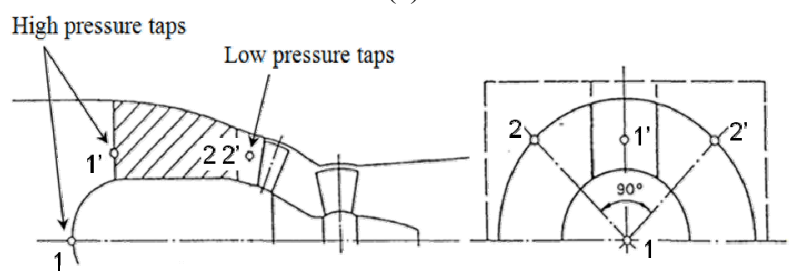

(b)

Fig. 1 Location of pressure taps in: (a) Winter-Kennedy method of discharge measurement through a Kaplan turbine equipped with a concrete half-spiral chamber; (b) differential pressure methods of discharge measurement through bulb turbine [1]. 
Both of these methods after their calibration using one of the primary methods (e.g., current meter method) and determination of the $K$ and $n$ constants can be used to measure the absolute value of the discharge through the turbine. In order to secure the highest possible correctness of the results obtained using these methods, the pressure difference should be measured using a high-accuracy transducer. Then, we can expect that the accuracy of measurements using the differential pressure method will be close to that of the method used for calibration.

Methods for determining the discharge based on the measurement of differential pressure, due to their characteristics, are recommended to use during optimization tests of the double-regulated turbines for measuring the relative discharge through the turbine. In order to obtain the correct result using these methods, it is important to construct the measurement systems properly (precise location of pressure taps, the tightness of the measuring system, and easy access to the equipment) and to use suitable measuring equipment (differential pressure transmitter of high class).

\subsection{The Current Meter Method}

The current meter method is one of the primary methods for measuring absolute discharge. It is based on the measurement of the local velocities using the current meters with well-known characteristics located at specific points of the measuring section.

These local velocities are then extrapolated to the entire flow cross-section and integrated to obtain the volumetric flow rate through the turbine. This method requires many conditions to be fulfilled, including in particular the selection of the appropriate cross-section of the flow system (away from the turbine and the obstacles which may deform the flow pattern) and the required number of properly installed current meters in the flow cross-section.

Because of optimization tests, there is no need to measure the absolute discharge, it is recommended to

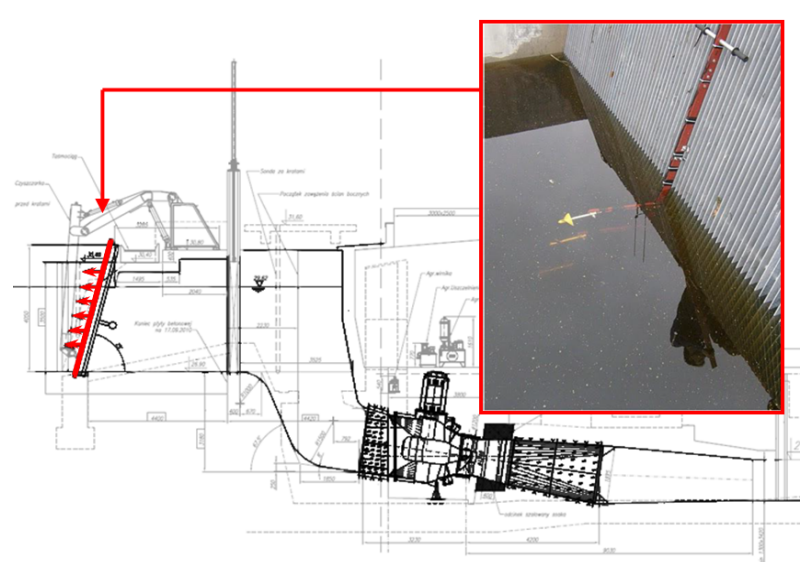

Fig. 2 Bulb turbine flow system with the cross section selected for installation of current meters used for measuring the relative discharge.

use the current meter index method. For this purpose, only one or several current meters located in the flow may be used (Fig. 2). Application of the index version of current meter method does not require complicated steps to make during the installation of the measuring system, and hence the out-of-operation time of the hydro unit needed for this stage is minimized. Using more than one current meter (if possible) reduces the uncertainty (scatter of measurements results are minimized).

\subsection{Comparison of Methods for Measuring the Relative Discharge through the Turbine}

The current meter method even in its index version is quite inconvenient to use because of the need for constructing special support structure for installation of the current meters, and placement in a particular (selected) cross-section often requires a longer out-of-operation time of the machine. This is very advantageous to select the cross-section which is characterized by minimal variation of the flow pattern. So it is recommended to install current meters as far as possible from the turbine and any obstacles in the flow system. Observance of this condition is not always possible. This is the case with tests carried out on low-head hydropower plants, which often are not equipped with sufficiently long, straight section of the inlet channel capable of producing a regular flow 
pattern (turbines with short intakes).

Application of methods for measuring the relative discharge, which are based on measurement of differential pressure in the two points between the flow systems of hydraulic turbine with double regulation, require correct installation of pressure taps. Investors should strive for a turbine with installed such measuring system because installing it in turbines already in operation requires high outlays and lost revenues associated with out-of-operation time of the machine. In some designs of turbines, such installations are not possible at all. Equipping the turbine with this installation and its maintenance is very beneficial, because it can be also used to measure the absolute discharge. Of course, it requires prior calibration using one of the primary methods (e.g., the current meter methods). The important point is that the results of absolute discharge measurements carried out by means of differential pressure method is characterized by the same uncertainty as the measurements obtained by method used for calibration.

Proper installation of the pressure taps has a significant impact on the obtained measurement results. Using the measurements of signals from poorly localized pressure taps or improperly prepared installation can lead to dramatically incorrect results. As a result, highly adverse effects of the optimization tests can be achieved. The example in Fig. 3 illustrates the impact of using the incorrect pressure taps to measure the pressure difference in the Winter-Kennedy method. The graph shows the relative propeller characteristics (for two different opening angles of the runner blades) of the Kaplan turbine measured during optimization test. The graph shows the points that differ significantly from the trend line made basing on points determined measuring pressure difference using the correct installation of the pressure taps. This discrepancy was caused by use an improper pressure taps to measure the pressure difference. Similar effects can occur at the untight (leaky) installation.

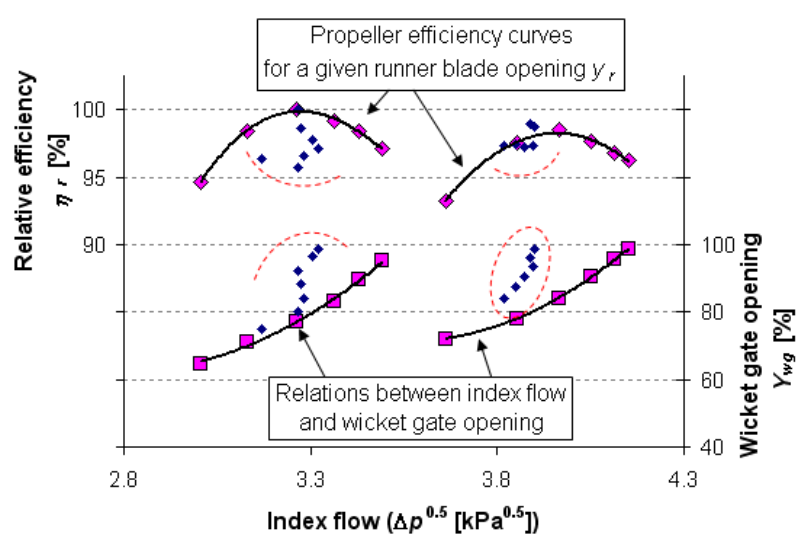

Fig. 3 Example of the propeller characteristics determined during optimization tests carried out using the differential pressure method basing on correct (points with trend lines) and incorrect pressure taps (points deviating from the trend lines marked by the dashed boundary line).

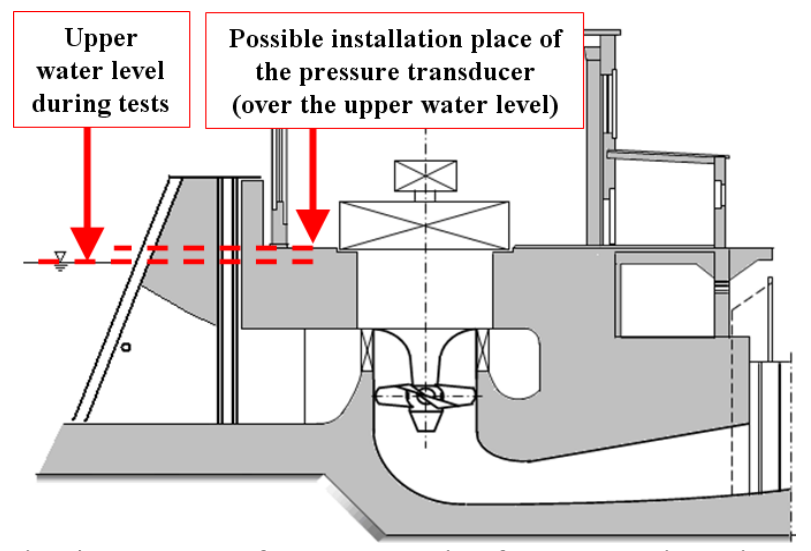

Fig. 4 Example of Kaplan turbine flow system in which a pressure transducer connected to a differential pressure system used in the Winter-Kennedy method for discharge measurement can be located only above the upper water level. A reasonable alternative method for measuring discharge in this case may be the current meter method.

Where the conditions (the design of the turbine flow system) do not allow or make it very difficult to apply the method based on differential pressure measurement, it becomes usually necessary to use the index current meter method. Fig. 4 shows an example of a flow system of the classic Kaplan turbine that makes it almost impossible to prepare proper installation of measuring system for differential pressure methods. In this case because of minor leaks and the fact that there was vacuum pressure in this system, it was impossible to remove air from the signal tubes. As a result, it was decided to use the index current meter method instead 
of the Winter-Kennedy method for measuring the relative discharge.

Generally, experience with the differential pressure methods for measuring the discharge through the turbine, both for optimization tests as well as to measure the absolute discharge (after prior calibration of the measuring system) has shown clearly its usefulness due to its ease of use and high rate of repeatability of results. It is worth to disseminate, especially in a small hydropower plants, because of its low costs and a very short out-of-operation time necessary for preparation of the tests.

\section{Examples of Optimization Tests of Double-Regulated Turbines}

\subsection{Measurable Effects of the Optimization Tests}

The example of the results of optimization tests carried out on the classic Kaplan turbine is presented in Fig. 5. The tests included the determination of nine propeller efficiency curves at a given head. For relative discharge measurement, the Winter-Kennedy method was used and the results are presented in relation to the index flow rate represented by square root of the pressure difference value $\Delta p_{\mathrm{W}-\mathrm{K}}^{0.5}$. Basing on the relative propeller curves of the index efficiency, the envelope efficiency curve was calculated and consequently the optimal cam dependence $y_{r b}=\mathrm{f}\left(Y_{w g}\right)$ was determined.

The differences between the optimal cam dependence and factory settings (initially set in the turbine governor) were highly significant. The consequence of these differences in this case is several percent lower efficiency of the machine operated at factory settings instead of optimal settings of the cam dependence. Further detailed analysis of these case showed that the operation of the turbine according to the non-optimal cam dependence leads to measurable production losses due to inefficient use of available hydropower potential.

\subsection{The Impact of the Head Changes on Optimal Operation of the Double-Regulated Turbine}

Very often, especially for low head hydrounits, the double-regulated turbines are operated at cam

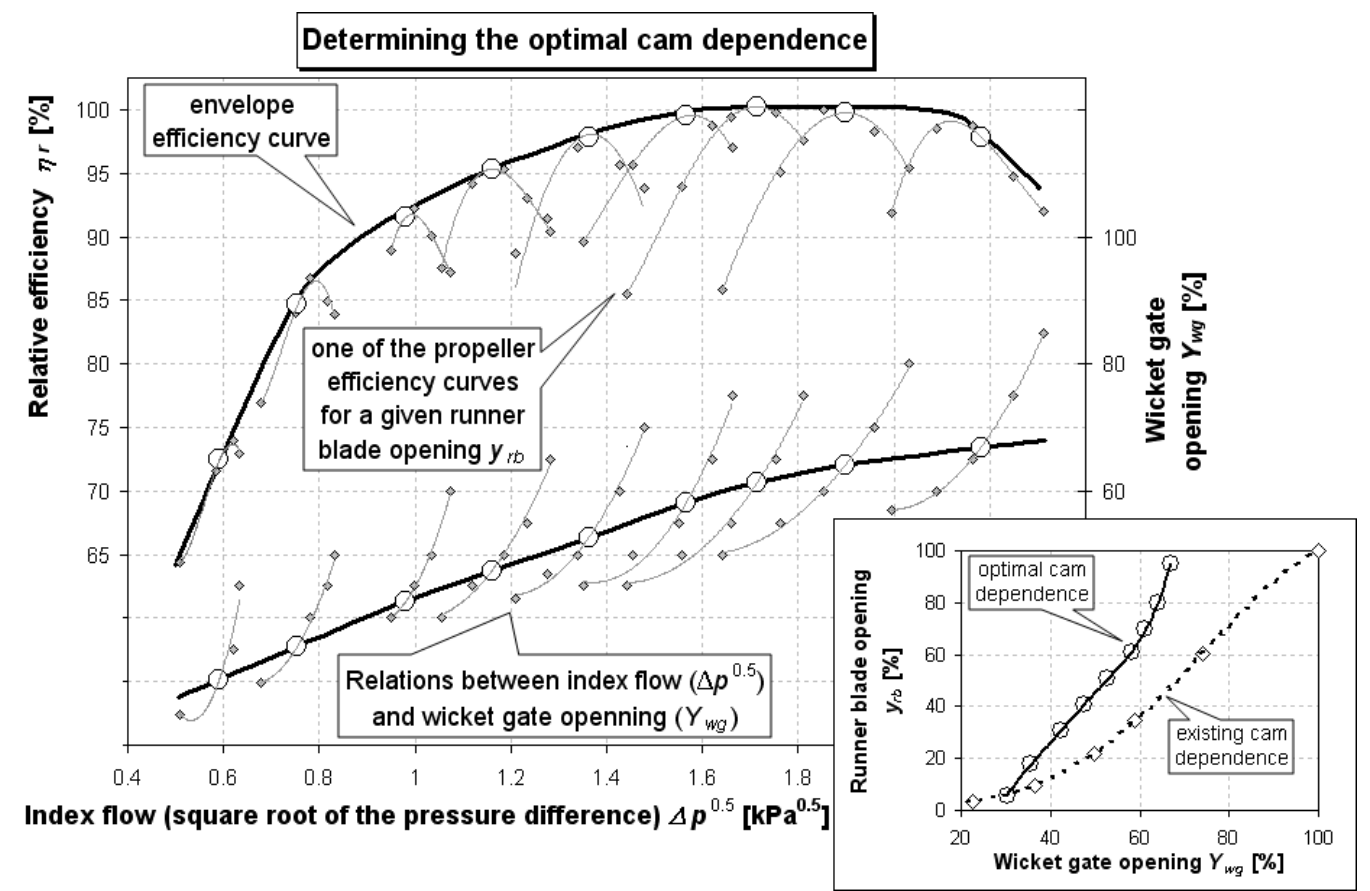

Fig. 5 Example of propeller curves with their envelope curve defined using the relative discharge measurement using Winter-Kennedy method (determined as the square root of pressure difference in the turbine spiral case $\Delta p^{0.5}$ ) and used to determine the optimal cam dependence $y_{r}=f\left(Y_{g}\right)$. 
dependences that do not take account of head changes. This is usually the consequence of decisions made during the design stage and avoiding the additional testing or numerical modeling by the turbine manufacturers. Investors generally agree to such solutions, assuming that there is no practical and sensible way of checking the effectiveness of sophisticated automatic systems of power control. Turbine manufacturers are taking advantage from the investors' lack of knowledge about the possibility to perform measurements using index methods, and confirm them that the designed cam dependence meets all the requirements for optimal operation of hydro unit. This approach is valid only for hydropower plants where there is little variation of disposable head. But if the head are changing in a wide range then using the cam dependence that disregards these changes can lead to significant loss of production. Fig. 6 shows an example that illustrates the positive effect obtained by replacing the cam dependence which does not take account of head changes with the optimal cam dependence determined respecting these changes. As a result of the optimization tests over $20 \%$ of relative efficiency increase at the maximum load has been achieved.

\subsection{The Impact of Modernizations on Optimal Operation of the Double-Regulated Turbine}

Very often the owners of hydropower plants operated for a long time decide to modernize the control systems of the hydro units. Modernization refers to hydraulic as well as electrical and mechanical parts of these systems. Usually, it means the exchange of the governors to those with modern hydraulic systems and components constructed using an advanced microprocessor technology. After such a deep modernization, the measurements to determine the optimal cam dependence are necessary. Withdrawing the optimization tests will certainly result in reduced utilization rate of disposable hydraulic potential which will lead to losses in electricity production and very

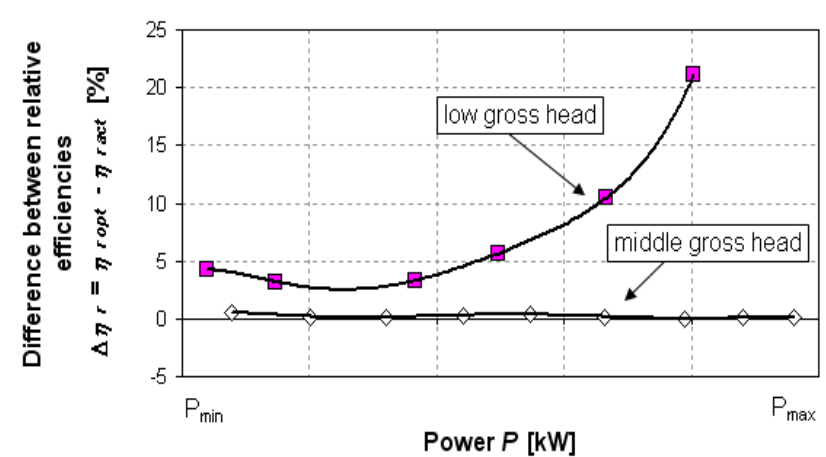

Fig. 6 Example of an increase in relative efficiency resulting from the replacement of existing cam dependence with optimal one taking account of the head changes.

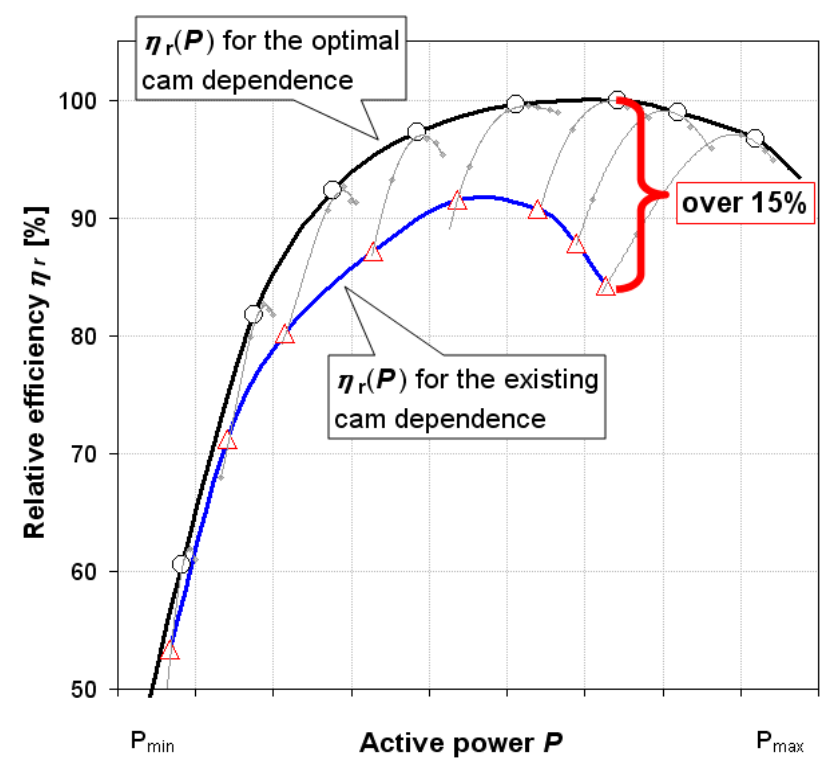

Fig. 7 Comparison of the relative efficiency curve before and after the modernization-the differences resulted from an incorrect translation of the control algorithm used in the turbine governor after changing the strokes of servo pistons in the regulation system.

often will be associated with the deterioration of the dynamic condition of the hydro unit. Fig. 7 shows the optimization test results of the classical Kaplan turbine obtained using the Winter-Kennedy method.

The graph compares the performance curves obtained at the optimal settings and at settings introduced immediately after the deep modernization of the hydro unit regulation system. In this case, modernization caused changes of strokes of servo pistons used for the wicket gates and runner blades openings, but this has not been included in the new control algorithm. This caused a loss of performance 
by over $15 \%$, and the inability to load the nominal power.

\section{Conclusions and Recommendations}

Analysis of the results of measurements carried out in the hydropower plants equipped with Kaplan-type turbines showed that in many cases the level of making use of disposable hydropower potential is highly unsatisfactory. The most common reason for this situation is operating of hydro units in non-optimal conditions, due to the use of improper relationship between wicket gates and runner blades openings, so called cam dependence.

Desisting from updating the optimal cam dependences by the hydropower plants owners is a consequence of underestimating the effects of operation with reduced efficiency of the hydrounits, and the relatively high costs of measurements of their performance tests.

Index methods used for measuring the energetic properties during the optimization tests allows to maximize the use of the available hydropower potential at relatively low costs of tests implementation and low costs associated with the required out-of-operation time of hydrounit required to prepare for these tests. These methods provide opportunities for dissemination of the optimization tests also for small hydropower plants, where the amount of production and the related revenue is too low to cover the cost of full research of energetic properties.

Low costs of optimization tests performed using methods described in the paper, especially the differential pressure method, results from the unnecessity of measuring the absolute discharge through the turbine but only its relative value.

Choosing the appropriate method for measuring the relative discharge through the turbine depends on the technical and hydrological conditions that occur on site. Wherever there is technically possible and economically justified, it is recommended to use methods based on the measurement of differential pressure between two points properly located in the turbine flow system. In comparison with other methods (especially with the index current meter method), the recommended method is characterized by:

- High certainty of the measured parameters due to applying precise and reliable components of the measurement system as well as a simple and clear setup of the system (no need for additional construction, easy to install a differential pressure transducer);

- Short duration of configuration of the measurement system, and thus minimizing the required out-of-operation time of the hydro unit, and also significantly shorter time needed for execution of measurements (comparing to other methods used in the measuring practice);

- Lack of the influence of flow disturbances connected with the characteristics of the flow system of the tested machine, provided the proper implementation and localization of pressure taps.

Equipping the turbine with the installations enabling to measure the pressure difference between two points precisely localized in the flow system, opens up the possibility for multiple measurements of absolute discharge (after calibrating this system using primary methods for measuring the absolute discharge through the turbine, e.g., using the current meter method) with high accuracy at low cost in relation to the implementation of the primary methods.

The scope of optimization tests depends largely on the nature of the turbine operation. If the turbine operates in conditions of significant head changes, it is recommended to relate the cam dependence from these changes. Desisting from taking account of head changes during optimization of the turbine can cause a drastic reduction in hydropower potential utilization rate, which translates directly into energy production and loss of revenue.

In case of upgrade or repair of turbine regulation systems, requiring remove of important elements of these systems, it is necessary to perform the optimization tests in order to reproduce the optimal 
cam dependence. Desisting from performing these tests may result in a significant reduction in the efficiency of the machine and sometimes significant deterioration in its dynamic states which may even lead to failures associated with serious consequences.

\section{Acknowledgments}

The paper was presented during the International Congress and Trade Fair on Small Hydropower HIDROENERGIA 2012 "Water-Powers-Life" held on 23-26 May, 2012 in Wroclaw, Poland.

\section{References}

[1] IEC 60041-1999: International Standard, Field acceptance tests to determine the hydraulic performance of hydraulic turbines, storage pumps and pump-turbines, The International Electrotechnical Commission, 1999.
[2] IEC 62006-2010: International Standard, Hydraulic machines-acceptance tests of Small Hydroelectric Installations, The International Electrotechnical Commission, 2010.

[3] Hydraulic Turbines and Pump-Turbines, Performance Test Codes, ASME PTC 18, 2002.

[4] M Kaniecki, Z. Krzemianowski, M. Banaszek, Computational fluid dynamics simulations of small capacity Kaplan turbines, Trans. of the Institute of Fluid-Flow Machinery 123 (2011) 71-84.

[5] Best Practice Catalog: Propeller/Kaplan Turbines, Mesa Associates, Inc. and Oak Ridge National Laboratory for U.S. Department of Energy, Hydropower Advancement Project, Rev. 1.0, June 12, 2011.

[6] L.H. Sheldon, Field testing and optimizing efficiency of hydro turbines, Water Power \& Dam Construction (1982) 22-28.

[7] B. Velenšek, Optimisation of Bulb and Kaplan turbine runners, in: Proc. of Int. Conf. on Hydropower, Waterpower, Portland, USA, 1987, pp. 1920-1929. 